\title{
Measurement range enlargement in Brillouin optical correlation- domain reflectometry based on chirp modulation scheme
}

\author{
Kohei Noda ${ }^{1,2}$, Heeyoung Lee ${ }^{3}$, Kentaro Nakamura ${ }^{1}$, and Yosuke Mizuno ${ }^{2 *}$ \\ 1 Institute of Innovative Research, Tokyo Institute of Technology, Yokohama 226-8503, Japan \\ 2 Faculty of Engineering, Yokohama National University, Yokohama 240-8501, Japan \\ 3 College of Engineering, Shibaura Institute of Technology, Tokyo 135-8548, Japan \\ *E-mail: mizuno-yosuke-rg@ynu.ac.jp
}

\begin{abstract}
Brillouin optical correlation-domain reflectometry (BOCDR) generally suffers from the trade-off relationship between the spatial resolution and the measurement range. In this work, we perform a proof-of-concept demonstration of a chirp modulation scheme to extend the measurement range of BOCDR. In this scheme, optical frequency is modulated using a chirp-shaped waveform. We show that, although the spatial resolution is slightly deteriorated, the measurement range and the range-to-resolution ratio can be relatively largely improved. In our preliminary experiment, the range-to-resolution ratio is 471 , which is approximately 3 times higher than that of a standard configuration, proving the effectiveness of this scheme.
\end{abstract}

Optical fiber sensing is one of the most promising techniques for monitoring aging infrastructures because of their various advantages over electric sensing, such as longrange measurement, immunity to electromagnetic noise, and the ease of embedding. Among numerous types of fiber-optic sensors, distributed strain and temperature sensors based on Brillouin scattering have been extensively studied. ${ }^{1)}$ To spatially resolve sensing locations, three major configurations, i.e., time- ${ }^{2-8)}$ frequency- ${ }^{9-12)}$ and correlationdomain techniques, ${ }^{13-19)}$ have been developed. A number of strategies for their performance improvement have been documented so far. ${ }^{3-5,7,8,10,11,14-17)}$ Needless to say, each configuration has its own advantages and disadvantages, and here we focus on the correlation-domain technique, ${ }^{13-19)}$ which has relatively high spatial resolution and random accessibility to sensing points.

The correlation-domain technique operates on the basis of synthesized optical coherence function $(\mathrm{SOCF}){ }^{20)}$ where distributed measurement is performed by modulating the optical frequency of a light source in a fiber-optic Mach-Zehnder interferometer. Since SOCF was first employed for two-end-access Brillouin measurement (currently referred to as Brillouin optical correlation-domain analysis 
(BOCDA), ${ }^{13)}$ a variety of configurations of distributed correlation-domain Brillouin sensors have been implemented. ${ }^{14-19)}$ They include a single-end-access configuration called Brillouin optical correlation-domain reflectometry (BOCDR). ${ }^{18,19)}$ To date, a lot of papers have been published aiming at the performance improvement of BOCDR, such as high-speed operation, ${ }^{21,22)}$ enhanced spatial resolution, ${ }^{23,24)}$ use of polymer optical fibers, ${ }^{25,26)}$ and system simplification. ${ }^{27,28)}$

Another trial for improving the performance of BOCDR is the elongation of its measurement range, which is reported to be in a trade-off relationship with spatial resolution. This aim has been achieved by two special schemes referred to as a "temporal gating scheme"29) and a "double modulation scheme". ${ }^{30)}$ In both schemes, a single specific correlation peak can be arbitrarily selected from multiple correlation peaks generated in the fiber under test (FUT). Combined use of these techniques has also been demonstrated. ${ }^{31)}$ A new scheme for extending the measurement range of BOCDR is of considerable interest, because it may lead to further performance improvement in the future.

In this work, as another scheme for improving the measurement range of BOCDR, we propose a "chirp modulation scheme", in which a chirp-shaped waveform is used to modulate the optical frequency. This waveform contains a variety of frequency components, each of which generates a correlation peak, and only their intersectional portion can operate as a sensing point. The other parts are mutually canceled out, resulting in an enlarged measurement range. As a preliminary experiment, we show that, when the modulation amplitude is constant, the range-to-resolution ratio can be increased from 168 to 471 . Some unique characteristics of this scheme are also discussed.

In most of the single-end-access Brillouin sensors, the laser output is divided into reference light and pump light. The pump light is injected to the FUT, and Brillouinscattered Stokes light is returned from each part of the FUT. The Stokes light is heterodyned with the reference light so that the Brillouin gain spectrum (BGS) can be extracted as a beat signal of the Stokes light and the reference light. The peak frequency of the BGS is down-shifted from that of the pump light, the amount of which is called Brillouin frequency shift (BFS). As BFS is in proportion to the strain and heat applied to the FUT, ${ }^{1)}$ strain and temperature information can be derived from the BFS measurement results. In BOCDR, to perform distributed sensing, the optical frequency of the laser output (continuous wave) is modulated to generate a correlation peak (corresponding to a sensing point) in the FUT. In a standard configuration of BOCDR, ${ }^{18,19)}$ the optical frequency is modulated with a sinusoidal waveform expressed by the following equation:

$$
f_{\sin }(t)=\Delta f \sin \left(2 \pi f_{m} t\right),
$$


where $f_{m}$ is the modulation frequency and $\Delta f$ is the modulation amplitude. With this configuration, the measurement range $D$ is restricted by the interval of the correlation peaks and given as

$$
D=\frac{c}{2 n f_{m}} \text {, }
$$

where $c$ is the light velocity in vacuum and $n$ is the core refractive index. The spatial resolution of BOCDR is generally determined by the signal level from a strained section against the noise structure generated from non-strained sections and given as ${ }^{13,19)}$

$$
\Delta z=\frac{c \Delta v_{B}}{2 \pi n f_{m} \Delta f},
$$

where $\Delta v_{B}$ is the Brillouin bandwidth. If we focus on $f_{\mathrm{m}}$, it is clear that $D$ and $\Delta z$ are in a trade-off relationship. Considering this situation, we often employ the ratio of $D$ to $\Delta z$, sometimes referred to as the number of effective measurement points, as a performance evaluation parameter, which is given as

$$
N=\frac{D}{\Delta z}=\frac{\pi \Delta f}{\Delta v_{\mathrm{B}}} .
$$

Considering that $\Delta f$ is generally limited to $\sim 5.5 \mathrm{GHz}$ (half of the BFS) in BOCDR ${ }^{18)}$ and that it is not easy to largely control $\Delta v_{\mathrm{B}}$, to mitigate the trade-off relationship and to enhance $N$, we need to employ some special schemes, such as a temporal gating scheme ${ }^{29)}$ and a double modulation scheme. ${ }^{30)}$ In both schemes, first, multiple correlation peaks are generated in the FUT, and subsequently, undesired peaks are suppressed by resolving the correlation peaks. In the temporal gating scheme, the Brillouin signal from a specific correlation peak is selectively detected using time differences of signal transmission; in contrast, in the double modulation scheme, optical frequency is doubly modulated by another frequency. Here, we newly propose a chirp modulation scheme, where the optical frequency is modulated by a chirp-shaped waveform.

A chirp-shaped waveform, which has been exploited in some sensing techniques including time-domain reflectometers, ${ }^{32,33)}$ generally contains a variety of frequencies. Each frequency component generates a correlation peak, and only their intersectional portion can operate as a sensing point; the other parts are mutually canceled out, resulting in an enlarged measurement range. Therefore, the measurement range can be calculated using Eq. (2) by substituting the repetition frequency of the chirp waveform to $f_{\mathrm{m}}$. However, it is difficult to theoretically evaluate the spatial resolution of this scheme; this point needs to be experimentally investigated.

The experimental setup of BOCDR based on the chirp-modulation scheme is basically the same as previously reported setups, ${ }^{18,19)}$ but we employed a recently developed 
externally modulated configuration, ${ }^{34,35)}$ as depicted in Fig. 1. With this configuration, optical frequency can be easily modulated by an arbitrary-shaped waveform without considering the transfer function of the laser. ${ }^{36)}$ The optical frequency of the laser output (NX8563LB, NEC) was modulated by a double-sideband modulator (DSBM), to which we applied microwave generated by a voltage-controlled oscillator (VCO; HMC733LC4B, Analog Devices). The output light involving lower and upper sidebands of the DSBM was input to an optical narrow bandpass filter (BVF-300CL, Alnair), and the upper sideband was selected as the desired frequency-modulated light. The chirpshaped modulation waveform used in this experiment is shown in Fig. 2. This waveform, generated by a function generator (FG; WF1948, NF Corporation), can be expressed by

$$
f_{\text {chirp }}(t)=\Delta f A_{t} \sin \left(2 \pi N_{p} f_{\text {rep }} t+\int m_{t} d t\right),
$$

where $f_{\text {rep }}$ is the repetition frequency, $N_{p}$ is the number of the peaks in the waveform, and $A_{t}$ (envelope function) and $m_{t}$ are given by

$$
\begin{gathered}
A_{t}=1+S\left(f_{\mathrm{rep}} t-\frac{1}{2}\right), \\
m_{t}=2 \pi m f_{\mathrm{rep}} t,
\end{gathered}
$$

where $S$ is the slope of the envelope, and $m$ is the modulation index of the chirp shape. In this experiment, we set $\Delta f=1.6 \mathrm{GHz}, S=1.33$ (for apodization, ${ }^{37,38)}$ which will be discussed below), $N_{p}=6$, and $m=5$. Note that these parameters are not theoretically optimized. We defined an effective starting frequency $f_{\text {start }}$ of the chirp waveform as a half of an inverse of the period between the first peak and the first dip in the waveform (Fig. 2). An effective stopping frequency $f_{\text {stop }}$ was also defined as a half of an inverse of the period between the first dip and the first peak (from the back). The values of $f_{\text {start }}$ and $f_{\text {stop }}$ were $10.42 f_{\text {rep }}$ and $3.17 f_{\text {rep }}$, respectively, where $f_{\text {rep }}$ was swept from $288.90 \mathrm{kHz}$ to $290.07 \mathrm{kHz}$. In this condition, the measurement range is calculated using Eq. (2) to be approximately $353 \mathrm{~m}$ (simply given by $f_{\text {rep }}$ ). The resolution bandwidth and the video bandwidth of an electrical spectrum analyzer (ESA) were $300 \mathrm{kHz}$ and $3 \mathrm{kHz}$, respectively. Averaging was performed 8 times in the ESA to obtain the BGS at a single sensing point.

The structure of the FUT used in the experiment is depicted in Fig. 3. A 308-m-long jacketed silica single-mode fiber (SMF) was connected to a $\sim 10-\mathrm{m}$-long unjacketed silica SMF (BFS $=10.86 \mathrm{GHz}$, pre-measured strain dependence coefficient of BFS $=448$ $\mathrm{MHz} / \%$ ) with an angled-physical-contact (APC) connector. A $0.33 \%$ strain was applied to a 1.0-m-long section of the shorter SMF. The strained length was varied from $1.0 \mathrm{~m}$ to $0.50 \mathrm{~m}$ with a step of $0.10 \mathrm{~m}$ to investigate the minimum length detectable with the chirp 
modulation scheme. A bending loss was applied near the distal end of the FUT to suppress the Fresnel reflection. The sensing point was scanned in the 303-316-m range (relative position from the APC connector between the jacketed SMF and the second-port pigtail of an optical circulator).

The BGS distributions measured when the strained length was $1.0 \mathrm{~m}, 0.8 \mathrm{~m}$, and 0.7 $\mathrm{m}$ are shown in Figs. 4(a), 4(b), and 4(c), respectively. The BFS in the strained section was not clear in Fig. 4(c), but it was clearly observed in Figs. 4(a) and 4(b). The base line of the BFS was $\sim 10.86 \mathrm{GHz}$, and the amount of the strain estimated by the BFS change was $\sim 0.33 \%$, which indicates that distributed BFS measurement was correctly performed in Figs. 4(a) and 4(b). Note that the BFS gap observed at $\sim 308 \mathrm{~m}$ was caused by the BFS difference between the two SMFs (fabricated by different manufacturers). The overall BFS fluctuations originated from the deteriorated signal-to-noise ratio caused by the use of external modulation. ${ }^{35)}$ Thus, the measurement range was experimentally shown to be longer in the chirp modulation scheme than those in sinusoidal modulation schemes with any $f_{m}$ value (ranging from $f_{\text {start }}$ to $f_{\text {stop }}$ ). As for the spatial resolution, it was estimated by this experiment to be $0.75 \pm 0.05 \mathrm{~m}$, which was deteriorated compared with that in a sinusoidal modulation scheme even with an $f_{m}$ value of $f_{\text {stop }}(=0.66 \mathrm{~m}$; when $\Delta f=1.6 \mathrm{GHz}$ ), as shown in Fig. 5. The deterioration in the spatial resolution probably originates from the influence of the apodization, i.e., the manipulated timeaveraged power spectrum of the light source. ${ }^{37,38)}$ This spectral apodization has been reported to suppress the noise structure (which is the beat signal caused by the outside of the major correlation peak in the measured BGS) and extend the strain dynamic range at the sacrifice of the reduced spatial resolution, and our scheme should not be an exception. Note that our scheme did not properly operate at $S=0$ (without apodization) because of the considerable noise structure and the resultant limited strain dynamic range; although the upper limit of the spatial resolution is nominally determined by $f_{\text {start }}$ (when $f_{\text {start }}>f_{\text {stop }}$ ), the actual resolution is determined by apodization, i.e., the non-zero $S$ value. Thus, in the chirp modulation scheme, the measurement range was extended while the spatial resolution was deteriorated compared to those in a conventional scheme. However, in this experiment, the range-to-resolution ratio $N$ was 471 , which was 2.8 times higher than 168 in the sinusoidal modulation scheme. Therefore, we can claim that the overall system performance was improved by the chirp modulation scheme. By optimizing each parameter of chirp modulation (including the change in the expression of a chirp waveform from Eq. (5) to another with a higher degree of freedom), further performance improvement should be feasible. Further study is required on this point, along with the effect of the combined use with the temporal gating and double modulation schemes. 
In conclusion, a chirp modulation scheme was newly proposed to mitigate the tradeoff relationship between the measurement range and spatial resolution in BOCDR. In this scheme, optical frequency is modulated using a chirp-shaped waveform. We experimentally show that the measurement range is largely extended but that the spatial resolution is slightly deteriorated. The obtained range-to-resolution ratio was 471 , which was approximately 3 times higher than that of a conventional sinusoidal modulation scheme, proving the effectiveness of the chirp modulation scheme. Combined use of this scheme and either (or both) of the temporal gating or/and double modulation schemes may be a future direction of further performance improvement of BOCDR. Remark that the chirp modulation scheme (as well as the other two schemes) is applicable not only to BOCDR but also to BOCDA and standard SOCF-OCDR. ${ }^{34,39,40)}$ Although the chirp modulation parameters need to be optimized, we believe that the proof-of-concept demonstration of this scheme will open up the potentials of correlation-domain distributed sensors in future.

\section{Acknowledgments}

This work was partly supported by JSPS KAKENHI Grant Numbers $17 \mathrm{H} 04930$ and 17J07226 and by research grants from the Noguchi Institute and the Telecommunications Advancement Foundation. 


\section{References}

1) G. P. Agrawal, Nonlinear Fiber Optics (Academic Press, San Diego, CA, 1995).

2) T. Horiguchi and M. Tateda, J. Lightwave Technol. 7, 1170 (1989).

3) D. Zhou, Y. Dong, B. Wang, C. Pang, D. Ba, H. Zhang, Z. Lu, H. Li, and X. Bao, Light: Sci. Appl. 7, 32 (2018).

4) Y. Peled, A. Motil, L. Yaron, and M. Tur, Opt. Express 19, 19845 (2011).

5) M. A. Soto, J. A. Ramírez, and L. Thévenaz, Nat. Commun. 7, 10870 (2016).

6) T. Kurashima, T. Horiguchi, H. Izumita, S. Furukawa, and Y. Koyamada, IEICE Trans. Commun. E76-B, 382 (1993).

7) A. Masoudi, M. Belal, and T. P. Newson, Opt. Lett. 38, 3312 (2013).

8) G. Tu, X. Zhang, Y. Zhang, Z. Ying, and L. Lv, Electron. Lett. 50, 1624 (2014).

9) D. Garus, K. Krebber, F. Schliep, and T. Gogolla, Opt. Lett. 21, 1402 (1996).

10) R. Bernini, A. Minardo, and L. Zeni, IEEE Photonics J. 4, 48 (2012).

11) D. Garus, T. Gogolla, K. Krebber, and F. Schliep, J. Lightwave Technol. 15, 654 (1997).

12) A. Minardo, R. Bernini, R. Ruiz-Lombera, J. Mirapeix, J. M. Lopez-Higuera, and L. Zeni, Opt. Express 24, 29994 (2016).

13) K. Hotate and T. Hasegawa, IEICE Trans. Electron. E83-C, 405 (2000).

14) K. Y. Song, M. Kishi, Z. He, and K. Hotate, Opt. Lett. 36, 2062 (2011).

15) A. López-Gil, S. Martin-Lopez, and M. Gonzalez-Herraez, Opt. Lett. 42, 3924 (2017).

16) Y. Okawa, R. K. Yamashita, M. Kishi, and K. Hotate, Opt. Express 28, 6981 (2020).

17) W. Zou, C. Jin, and J. Chen, Appl. Phys. Express 5, 082503 (2012).

18) Y. Mizuno, W. Zou, Z. He, and K. Hotate, Opt. Express 16, 12148 (2008).

19) Y. Mizuno, W. Zou, Z. He, and K. Hotate, J. Lightwave Technol. 28, 3300 (2010).

20) K. Hotate and Z. He, J. Lightwave Technol. 24, 2541 (2006).

21) Y. Mizuno, N. Hayashi, H. Fukuda, K. Y. Song, and K. Nakamura, Light: Sci. Appl. 5, e16184 (2016).

22) H. Lee, N. Hayashi, Y. Mizuno, and K. Nakamura, IEEE Photonics J. 8, 6802807 (2016).

23) Y. Mizuno, Z. He, and K. Hotate, Opt. Commun. 283, 2438 (2010).

24) H. Lee, Y. Mizuno, and K. Nakamura, Jpn. J. Appl. Phys. 57, 020303 (2018).

25) N. Hayashi, Y. Mizuno, and K. Nakamura, J. Lightwave Technol. 32, 3999 (2014).

26) Y. Mizuno, H. Lee, N. Hayashi, and K. Nakamura, Opt. Lett. 44, 2097 (2019).

27) Y. Mizuno, G. Han, K. Noda, H. Lee, and K. Nakamura, Electron. Lett. 55, 754 (2019).

28) Y. Mizuno, N. Motoishi, K. Noda, H. Lee, and K. Nakamura, Appl. Phys. Express 13, 052001 (2020). 
29) Y. Mizuno, Z. He, and K. Hotate, Opt. Express 17, 9040 (2009).

30) Y. Mizuno, Z. He, and K. Hotate, Opt. Express 18, 5926 (2010).

31) R. Shimizu, M. Kishi, and K. Hotate, Proc. SPIE 10323, 1032390 (2017).

32) J. Pastor-Graells, H. F. Martins, A. Garcia-Ruiz, S. Martin-Lopez, and M. Gonzalez-Herraez, Opt. Express 24, 13121 (2016).

33) A. E. Alekseev, V. S. Vdovenko, B. G. Gorshkov, V. T. Potapov, and D. E. Simikin, Laser Phys. 26, 035101 (2016).

34) K. Noda, G. Han, H. Lee, Y. Mizuno, and K. Nakamura, Appl. Phys. Express 12, 022005 (2019).

35) K. Noda, H. Lee, Y. Mizuno, and K. Nakamura, Jpn. J. Appl. Phys. 58, 068004 (2019).

36) K. Y. Song and S. Yang, Opt. Express 18, 24012 (2010).

37) K. Y. Song, Z. He, and K. Hotate, Opt. Express 14, 4256 (2006).

38) K. Y. Song, Z. He, and K. Hotate, J. Lightwave Technol. 25, 1238 (2007).

39) K. Hotate, M. Enyama, S. Yamashita, and Y. Nasu, Meas. Sci. Technol. 15, 148 (2004).

40) M. Shizuka, S. Shimada, N. Hayashi, Y. Mizuno, and K. Nakamura, Appl. Phys. Express 9, 032702 (2016). 


\section{Figure Captions}

Fig. 1. Experimental setup of externally modulated BOCDR for chirp modulation scheme. DSBM: double-sideband modulator, EDFA: erbium-doped fiber amplifier, ESA: electrical spectrum analyzer, FG: function generator, FUT: fiber under test, PD: photodiode, PSCR: polarization scrambler, VCO: voltage-controlled oscillator.

Fig. 2. Chirp-shaped waveform used to modulate optical frequency in the experiment.

Fig. 3. Structure of the FUT measured in the experiment.

Fig. 4. BGS distributions measured when the strained length was (a) $1.0 \mathrm{~m}$, (b) $0.8 \mathrm{~m}$, and (c) $0.7 \mathrm{~m}$.

Fig. 5. Measurement range vs. spatial resolution. Comparison between the chirp modulation and the sinusoidal modulation schemes. 


\section{Figures}

Fig. 1

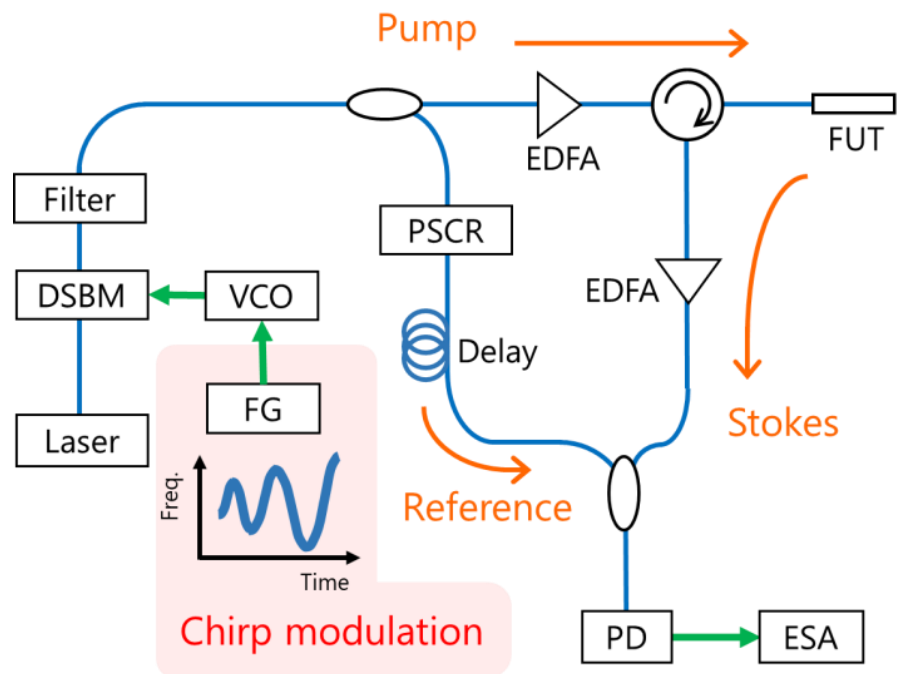

Fig. 2

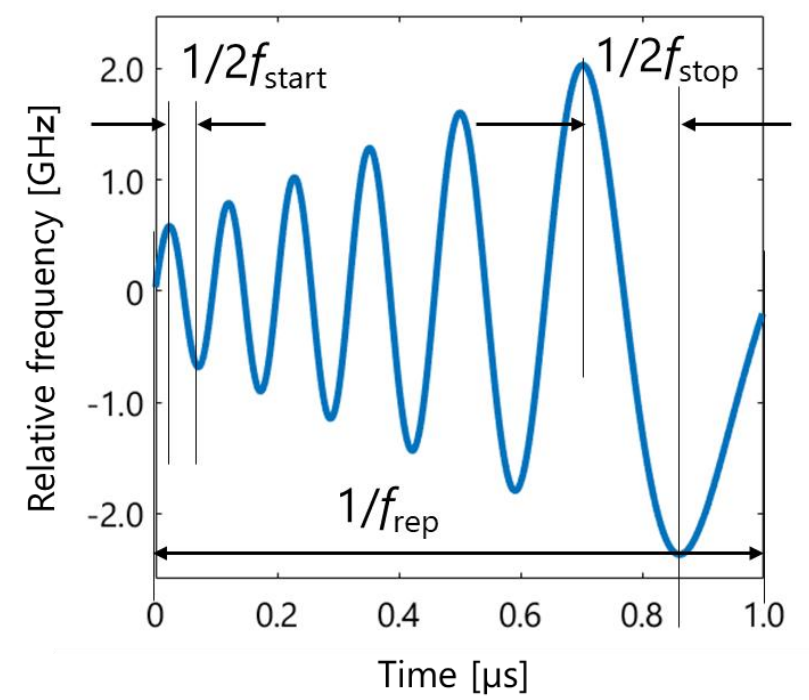


Fig. 3

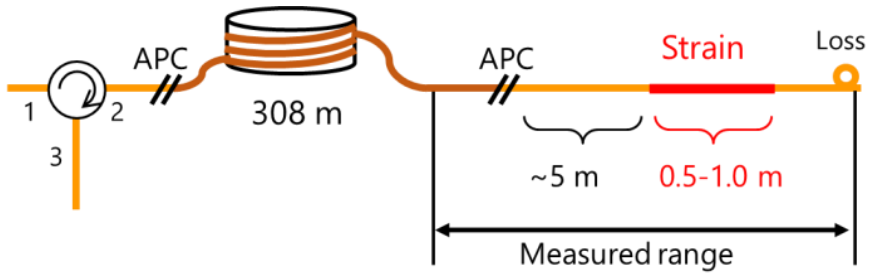

Fig. 4

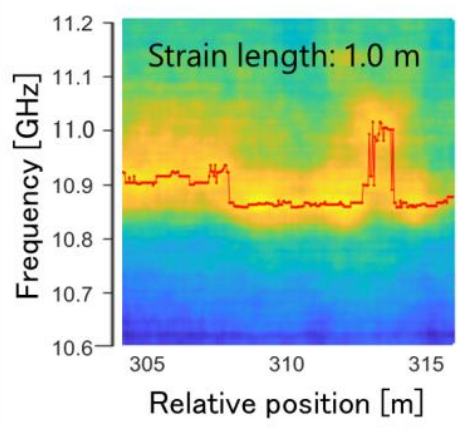

(a)

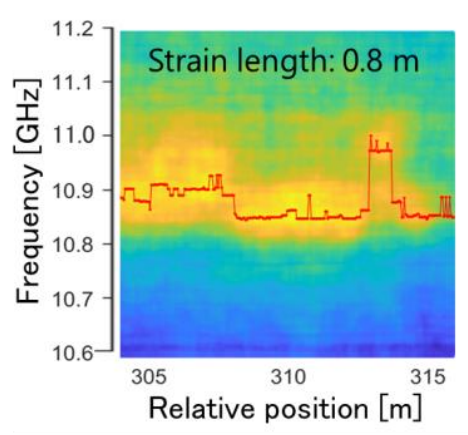

(b)

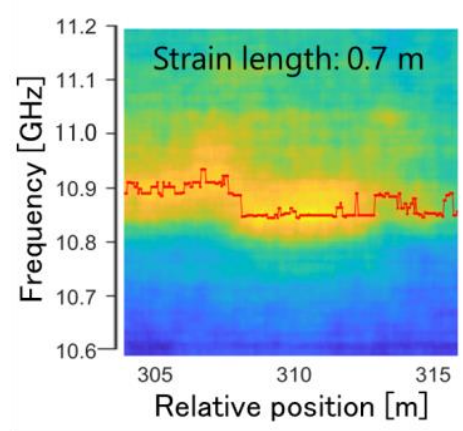

(c)

Fig. 5

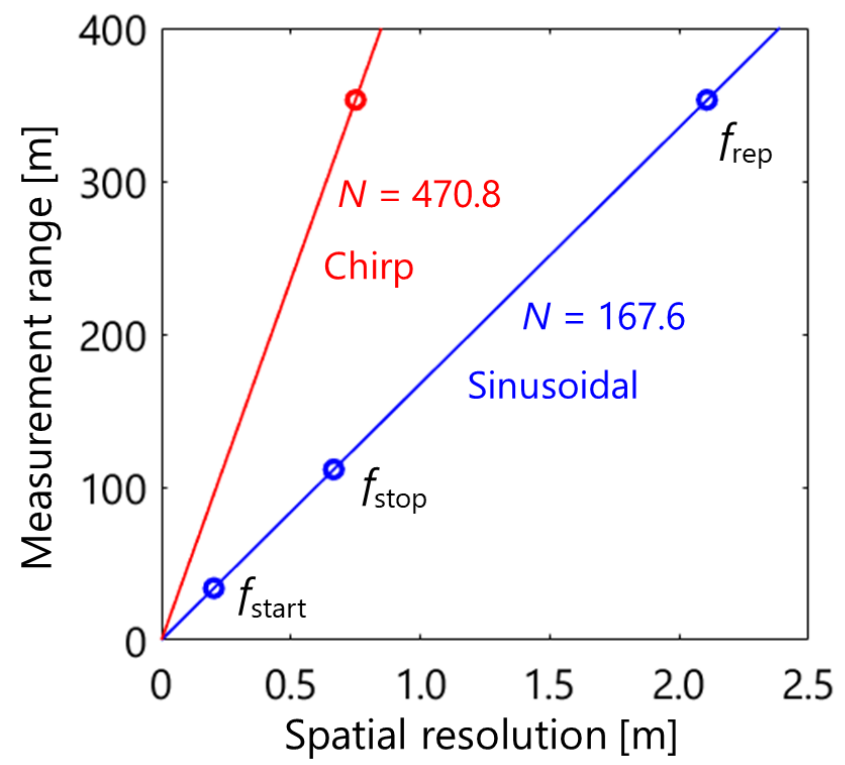

